\title{
Numerical Fourier Transforms in One, Two, and Three Dimensions for Liquid State Calculations
}

F. LADO

Department of Physics, North Carolina State University, Raleigh, North Carolina 27607

Received March 30, 1971

\begin{abstract}
When a Fourier transform (FT) is to be numerically computed and subsequently numerically inverted, perhaps repetitively, it is desirable that the algorithm used to accomplish this should maintain the orthogonal nature of the Fourier expansion. Algorithms with this property are derived here for the FT of central functions in one, two, and three dimensions. These rules for mechanical quadrature are similar to the trapezoidal rule, but with intervals determined by the zeros of the orthogonal basis functions. A numerical test using Gaussians shows a high accuracy in the computed transforms. The procedure can be extended to other Fourier-Bessel transforms.
\end{abstract}

\section{INTRODUCTION}

A problem often encountered in the equilibrium theory of fluids is the numerical solution of a convolution-type integral equation of the form

$$
F(r)=C(r)+\rho \int d \mathbf{r}^{\prime} F\left(r^{\prime}\right) C\left(\left|\mathbf{r}-\mathbf{r}^{\prime}\right|\right),
$$

where $C$ is a given functional of $F$. Such nonlinear equations are conveniently solved iteratively by means of Fourier transforms, which effect a deconvolution of the integral in (1). Let a tilde designate a Fourier transform, so that, for a function $F(r)$, we have

$$
\tilde{F}(\mathbf{k})=\int d \mathbf{r} F(\mathbf{r}) e^{-i \mathbf{k} \cdot \mathbf{r}},
$$

and similarly for $\tilde{C}(k)$. Applying this transformation to (1) then yields

$$
\tilde{F}(k)=\tilde{C}(k) /[1-\rho \tilde{C}(k)],
$$

and the desired solution after one iteration is finally obtained by Fourier inversion of (3), using the fact that

$$
F(\mathbf{r})=(2 \pi)^{-d} \int d \mathbf{k} \tilde{F}(\mathbf{k}) e^{i \mathbf{k} \cdot \mathbf{r}},
$$


where both $\mathbf{r}$ and $\mathbf{k}$ are $d$-dimensional vectors. An essential ingredient of this procedure is the orthogonality of the exponential functions, expressed by the equation

$$
\int d \mathbf{k} e^{i k \cdot(\mathbf{r}-\mathbf{r})}=(2 \pi)^{d} \delta\left(\mathbf{r}-\mathbf{r}^{\prime}\right) .
$$

If the solutions are to be obtained by numerical methods, it is therefore desirable to employ an algorithm for the transform calculation that maintains the orthogonality of the basis functions, so that the reciprocal relation between a function and its transform is thereby maintained in the numerical calculation. In this paper, we describe such algorithms for Fourier transforms in one, two, and three dimensions. These will be useful not only in equilibrium calculations, as sketched above, but also in nonequilibrium problems where it is desired to compute the one-dimensional transform of a time-dependent function.

A simplifying property shared by functions $F(\mathbf{r})$ defined for a fluid is the dependence of $F$ on only the magnitude $r$ of the vector $\mathbf{r}$. When this is the case, the transform $\tilde{F}$ becomes similarly a function of the magnitude $k$ alone. We will restrict the present discussion to such central functions, for which Eqs. (2), (4), and (5) take on the following simpler forms:

$$
\begin{gathered}
F(r)=\frac{1}{\pi} \int_{0}^{\infty} d k \tilde{F}(k) \cos k r, \\
\tilde{F}(k)=2 \int_{0}^{\infty} d r F(r) \cos k r, \\
\int_{0}^{\infty} d k \cos k r \cos k r^{\prime}=\frac{1}{2} \pi \delta\left(r-r^{\prime}\right)
\end{gathered}
$$

for $d=1$;

$$
\begin{gathered}
F(r)=\frac{1}{2 \pi} \int_{0}^{\infty} d k k \tilde{F}(k) J_{0}(k r), \\
F(k)=2 \pi \int_{0}^{\infty} d r r F(r) J_{0}(k r), \\
\int_{0}^{\infty} d k k J_{0}(k r) J_{0}\left(k r^{\prime}\right)=\frac{1}{r} \delta\left(r-r^{\prime}\right)
\end{gathered}
$$

for $d=2$; and

$$
\begin{gathered}
F(r)=\frac{1}{2 \pi^{2} r} \int_{0}^{\infty} d k k \tilde{F}(k) \sin k r, \\
\tilde{F}(k)=\frac{4 \pi}{k} \int_{0}^{\infty} d r r F(r) \sin k r, \\
\int_{0}^{\infty} d k \sin k r \sin k r^{\prime}=\frac{1}{2} \pi \delta\left(r-r^{\prime}\right)
\end{gathered}
$$


for $d=3$. In Eqs. (6b)-(8b), $J_{0}(x)$ is the zeroth-order Bessel function of the first kind which, with the representation [1]

$$
J_{0}(x)=(2 \pi)^{-1} \int_{0}^{2 \pi} d \theta \exp (i x \cos \theta),
$$

appears upon integration over the polar angle in two dimensions.

The difficulty with the numerical evaluation of the remaining one-dimensional integrals is well known. Referring to (6a), for example, one finds that for large values of $r$ the oscillations in $k$ of $\cos k r$ are so rapid that the usual quadrature rules, based on a small sample of integrand values at discrete points, break down. Various schemes have been proposed to overcome this difficulty. Filon [2] suggested a rule based on fitting just $\tilde{F}(k)$ [still referring to (6a)] by a quadratic polynomial between discrete points and evaluating the resulting integrals exactly. This algorithm, though accurate, does not preserve the orthogonal nature of the Fourier expansion, so that, in going back and forth between a function and its transform, an additional source of inaccuracy is introduced into the numerical calculation. More recently, the fast Fourier transform method [3] has been reintroduced and generalized by Cooley and Tukey [4]. As the name implies, this is a particularly efficient numerical procedure which, in addition, maintains orthogonality. In essence, however, this algorithm, which is most often used with the number of intervals $N$ equal to a power of 2, is a device for the rapid evaluation of a given trigonometric sum and not a general rule for determining the form of the sum initially. Thus, it could be applied to the sums obtained in Section IV for the threedimensional transforms, or, with some modifications, to the one-dimensional case of Section II. Since the technique makes essential use of the periodicity of the trigonometric functions, however, it is not applicable to the Hankel transforms of (6b) and (7b), whose discrete version is considered in Section III, for these contain the almost-periodic Bessel function.

As already indicated, the numerical versions of the Fourier transforms (FT) in one, two, and three dimensions are derived in Section II, III, and IV, respectively. These rules for mechanical quadratures to replace the integrals in (6a)-(8c) all maintain the orthogonal nature of the original Fourier expansions and are obtained through a common method which may be extended to other transforms. The results of a numerical check of these algorithms, employing a Gaussian test function for which the exact transform (also Gaussians) can be obtained, are summarized in Section V.

\section{Numerical FT in ONe Dimension}

There are two steps necessary to convert Eqs. (6a)-(8a) into forms suitable for mechanical quadrature: (1) the infinite limits must be replaced by finite ones, and 
(2) the integrals must be replaced by sums. As will be seen, these steps are not independent.

We will proceed in two stages. Both the functions $F(r)$ and $\tilde{F}(k)$ become vanishingly small as their arguments become infinitely large. Suppose, first, that $F(r)$ vanishes exactly for $r \geqslant R$. This boundary condition then requires that each term in the Fourier expansion of $F(r)$ vanish at $r=R$; i.e., we must have

$$
\cos k R=0,
$$

or

$$
k_{j} R=(2 j-1) \pi / 2, \quad j=1,2,3, \ldots .
$$

The allowed values of the wave number $k$ are then

$$
k_{j}=\left(j-\frac{1}{2}\right) \pi / R, \quad j=1,2,3, \ldots,
$$

so that (6a) reverts to a series form,

$$
F(r)=\frac{1}{\pi} \sum_{j=1}^{\infty} w_{j} \hat{F}\left(k_{j}\right) \cos k_{j} r,
$$

where the weights $w_{j}$ are chosen so that the form of (7a) is maintained:

$$
\begin{aligned}
\hat{F}\left(k_{j}\right) & =2 \int_{0}^{R} d r F(r) \cos k_{j} r \\
& =\frac{2}{\pi} \sum_{\ell=1}^{\infty} w_{\ell} \hat{F}\left(k_{l}\right) \int_{0}^{R} d r \cos k_{j} r \cos k_{l} r \\
& =R w_{j} \hat{F}\left(k_{j}\right) / \pi .
\end{aligned}
$$

Here we have used

$$
\int_{0}^{R} d r \cos k_{j} r \cos k_{\ell} r=\frac{1}{2} R \delta_{j \ell}
$$

Thus, the weights

$$
w_{j}=\pi / R=\Delta k \equiv k_{j+1}-k_{j}
$$

are all equal and we have the intermediate result that (6a) and (7a) become

$$
\begin{aligned}
& F(r)=\frac{1}{\pi} \sum_{j=1}^{\infty} \Delta k \tilde{F}\left(k_{j}\right) \cos k_{j} r, \\
& \tilde{F}\left(k_{j}\right)=2 \int_{0}^{R} d r F(r) \cos k_{j} r
\end{aligned}
$$

when $F(r)$ terminates at $R$. 
Now suppose further that $\tilde{F}\left(k_{j}\right)$ vanishes for $k_{j} \geqslant k_{N}=\left(N-\frac{1}{2}\right) \pi / R$. Then, in a similar fashion, we must have

$$
\cos k_{N} r=0,
$$

or

$$
k_{N} r_{i}=(2 i-1) \pi / 2,
$$

so that the allowed values of $r$ are

$$
r_{i}=\left(i-\frac{1}{2}\right) R /\left(N-\frac{1}{2}\right), \quad i=1,2,3, \ldots, N .
$$

We now write (18) as a sum over these allowed values, with weights $w_{i}{ }^{\prime}$,

$$
\tilde{F}\left(k_{j}\right)=2 \sum_{i=1}^{N} w_{i}^{\prime} F\left(r_{i}\right) \cos k_{j} r_{i},
$$

so that (17) now reads

$$
\begin{aligned}
F\left(r_{i}\right) & =\frac{1}{\pi} \sum_{j=1}^{N} \Delta k \tilde{F}\left(k_{j}\right) \cos k_{j} r_{i} \\
& =\frac{2 \Delta k}{\pi} \sum_{\ell=1}^{N} w_{\ell}^{\prime} F\left(r_{\ell}\right) \sum_{j=1}^{N} \cos k_{j} r_{i} \cos k_{j} r_{\ell} \\
& =\Delta k(2 N-1) w_{i}^{\prime} F\left(r_{i}\right) / 2 \pi .
\end{aligned}
$$

Here we have used the discrete orthogonality condition

$$
\sum_{j=1}^{N} \cos k_{j} r_{i} \cos k_{j} r_{\ell}=1(2 N-1) \delta_{i \ell},
$$

which is proved in Appendix A. Thus the weights

$$
w_{i}^{\prime}=R /\left(N-\frac{1}{2}\right)=\Delta r \equiv r_{i+1}-r_{i}
$$

are again equal. This completes the required changes.

Summarizing, we have found for the discrete versions of Eqs. (6a)-(8a),

$$
\begin{gathered}
F\left(r_{i}\right)=\frac{1}{\pi} \sum_{j=1}^{N-1} \Delta k \tilde{F}\left(k_{j}\right) \cos k_{j} r_{i}, \\
\tilde{F}\left(k_{j}\right)=2 \sum_{i=1}^{N-1} \Delta r F\left(r_{i}\right) \cos k_{j} r_{i}, \\
\sum_{j=1}^{N-1} \Delta k \cos k_{j} r_{i} \cos k_{j} r_{\ell}=\frac{\pi}{2} \frac{\delta_{i \ell}}{\Delta r},
\end{gathered}
$$


with

$$
\begin{aligned}
r_{i} & =\left(i-\frac{1}{2}\right) \Delta r, & \Delta r & =R /\left(N-\frac{1}{2}\right), \\
k_{j} & =\left(j-\frac{1}{2}\right) \Delta k, & \Delta k & =\pi / R .
\end{aligned}
$$

With this prescription a free choice of range $R$ and number of intervals $N$ determines the remaining parameters. Further, each of the discrete functions $F\left(r_{i}\right)$ and $\tilde{F}\left(k_{j}\right)$ is, through (28), the discrete transform of the other, in a manner completely analogous to the original continuous version. We remark finally that the upper limits of the sums in (26)-(28) have been put at $N-1$ since in each case the $N$-th term vanishes. These sums are evidently similar to a trapezoidal rule with equal intervals, the intervals being determined by the roots of $\cos x$.

\section{Numerical FT in Two Dimensions}

To find the discrete counterparts of Eqs. (6b)-(8b), we proceed as in the previous one-dimensional case. First, assume that $F(r)$ vanishes for $r \geqslant R$. This requires that $k$ satisfy

$$
J_{0}(k R)=0,
$$

or

$$
k_{j}=\mu_{j} / R, \quad j=1,2,3, \ldots,
$$

where $\mu_{j}$ is the $j$-th positive root of $J_{0}(x)$. The integral over $k$ now becomes a discrete sum, which we write as

$$
F(r)=\frac{1}{2 \pi} \sum_{j=1}^{\infty} w_{j} k_{j} \tilde{F}\left(k_{j}\right) J_{0}\left(k_{j} r\right),
$$

where $w_{j}$ is again such as to preserve the form of the Fourier inversion:

$$
\begin{aligned}
\tilde{F}\left(k_{j}\right) & =2 \pi \int_{0}^{R} d r r F(r) J_{0}\left(k_{j} r\right) \\
& =\sum_{\ell=1}^{\infty} w_{\ell} k_{\ell} \tilde{F}\left(k_{\ell}\right) \int_{0}^{R} d r r J_{0}\left(k_{j} r\right) J_{0}\left(k_{\ell} r\right) \\
& =\frac{1}{2} w_{j} R^{2}\left[J_{0}^{\prime}\left(k_{j} R\right)\right]^{2} k_{j} F\left(k_{j}\right) .
\end{aligned}
$$

Here we have used the orthogonality condition [5]

$$
\int_{0}^{R} d r r J_{0}\left(k_{j} r\right) J_{0}\left(k_{\ell} r\right)=\frac{1}{2} R^{2}\left[J_{0}^{\prime}\left(\mu_{j}\right)\right]^{2} \delta_{j t} .
$$


Thus the required weights are in this case unequal,

$$
w_{j}=\frac{2}{R^{2} k_{j}\left[J_{0}^{\prime}\left(k_{j} R\right)\right]^{2}},
$$

and we find that the intermediate result is an ordinary Fourier-Bessel expansion [6]

$$
\begin{aligned}
F(r) & =\frac{1}{\pi R^{2}} \sum_{j=1}^{\infty} f\left(k_{j}\right) \frac{J_{0}\left(k_{j} r\right)}{\left[J_{0}^{\prime}\left(k_{j} R\right)\right]^{2}}, \\
F\left(k_{j}\right) & =2 \pi \int_{0}^{R} d r r F(r) J_{0}\left(k_{j} r\right) .
\end{aligned}
$$

Now again suppose further that $f\left(k_{j}\right)$ is negligible for $k_{j} \geqslant k_{N}=\mu_{N} / R$, leading to the requirement

$$
J_{0}\left(k_{N} r\right)=0
$$

or

$$
r_{i}=\mu_{i} / k_{N}=\mu_{i} R / \mu_{N}, \quad i=1,2, \ldots, N
$$

With this set of permitted values (38) becomes

$$
\hat{F}\left(k_{j}\right)=2 \pi \sum_{i=1}^{N} w_{i}^{\prime} r_{i} F\left(r_{i}\right) J_{0}\left(k_{j} r_{i}\right),
$$

and hence we have

$$
\begin{aligned}
F\left(r_{i}\right) & =\frac{1}{\pi R^{2}} \sum_{j=1}^{N} \hat{F}\left(k_{j}\right) \frac{J_{0}\left(k_{j} r_{i}\right)}{\left[J_{0}^{\prime}\left(k_{j} R\right)\right]^{2}} \\
& =\frac{2}{R^{2}} \sum_{\ell=1}^{N} w_{\ell}^{\prime} r_{\ell} F\left(r_{\ell}\right) \sum_{j=1}^{N} \frac{J_{0}\left(k_{j} r_{i}\right) J_{0}\left(k_{j} r_{\ell}\right)}{\left[J_{0}^{\prime}\left(k_{j} R\right)\right]^{2}} \\
& =\frac{1}{2} w_{i}^{\prime} K^{2}\left[J_{0}^{\prime}\left(K r_{i}\right)\right]^{2} r_{i} F\left(r_{i}\right),
\end{aligned}
$$

whence

$$
w_{i}^{\prime}=\frac{2}{K^{2} r_{i}\left[J_{0}^{\prime}\left(K r_{i}\right)\right]^{2}},
$$

where we have put

$$
K \equiv k_{N} .
$$


In obtaining (42), we have used an (approximate) orthogonality relation established in Appendix $B$,

$$
\sum_{j=1}^{N} \frac{J_{0}\left(k_{j} r_{i}\right) J_{0}\left(k_{j} r_{\ell}\right)}{\left[J_{0}^{\prime}\left(k_{j} R\right)\right]^{2}}=\frac{1}{4} \mu_{N}^{2}\left[J_{0}^{\prime}\left(K r_{i}\right)\right]^{2} \delta_{i \ell}
$$

Summarizing these results, we have finally for the discrete version of Eqs. (6b)(8b):

$$
\begin{gathered}
F\left(r_{i}\right)=\frac{1}{\pi R^{2}} \sum_{j=1}^{N-1} \tilde{F}\left(k_{j}\right) \frac{J_{0}\left(k_{j} r_{i}\right)}{\left[J_{0}^{\prime}\left(k_{j} R\right)\right]^{2}}, \\
\tilde{F}\left(k_{j}\right)=\frac{4 \pi}{K^{2}} \sum_{i=1}^{N-1} F\left(r_{i}\right) \frac{J_{0}\left(k_{j} r_{i}\right)}{\left[J_{0}^{\prime}\left(K r_{i}\right)\right]^{2}}, \\
\sum_{j=1}^{N-1} \frac{J_{0}\left(k_{j} r_{i}\right) J_{0}\left(k_{j} r_{\ell}\right)}{\left[J_{0}^{\prime}\left(k_{j} R\right)\right]^{2}}=\frac{R^{2} K^{2}}{4}\left[J_{0}^{\prime}\left(K r_{i}\right)\right]^{2} \delta_{i \ell},
\end{gathered}
$$

with

$$
\begin{aligned}
& r_{i}=\mu_{i} R / \mu_{N} \\
& k_{j}=\mu_{j} / R,
\end{aligned}
$$

where the $\mu_{i}$ are the positive roots of $J_{0}(x)$. If we put

$$
\begin{aligned}
\Delta r_{i} & \equiv \frac{2}{K^{2} r_{i}\left[J_{0}^{\prime}\left(K r_{i}\right)\right]^{2}}, \\
\Delta k_{j} & \equiv \frac{2}{R^{2} k_{j}\left[J_{0}^{\prime}\left(k_{j} R\right)\right]^{2}},
\end{aligned}
$$

Eqs. (46)-(48) take on the appearance of a trapezoidal rule with unequal intervals:

$$
\begin{aligned}
& F\left(r_{i}\right)=\frac{1}{2 \pi} \sum_{j=1}^{N-1} \Delta k_{j} k_{j} \tilde{F}\left(k_{j}\right) J_{0}\left(k_{j} r_{i}\right), \\
& \tilde{F}\left(k_{j}\right)=2 \pi \sum_{i=1}^{N-1} \Delta r_{i} r_{i} F\left(r_{i}\right) J_{0}\left(k_{j} r_{i}\right), \\
& \sum_{j=1}^{N-1} \Delta k_{j} k_{j} J_{0}\left(k_{j} r_{i}\right) J_{0}\left(k_{j} r_{\ell}\right)=\frac{1}{r_{i}} \frac{\delta_{i \ell}}{\Delta r_{i}} .
\end{aligned}
$$


Note that, using the asymptotic forms [7]

$$
\begin{aligned}
\mu_{j} & \sim\left(j-\frac{1}{4}\right) \pi, \\
J_{0}{ }^{\prime}(x) & \sim(2 / \pi x)^{1 / 2} \sin \left(x-\frac{1}{4} \pi\right),
\end{aligned}
$$

we have, from (51) and (52),

$$
\begin{aligned}
& \Delta r_{i} \sim R /\left(N-\frac{1}{4}\right), \\
& \Delta k_{i} \sim \pi / R,
\end{aligned}
$$

which may be compared with Eqs. (29), (30) and (63), (64). Finally, we note that, as in the previous case, $R$ and $N$ are to be freely chosen, all other parameters of the algorithm following from this choice.

\section{Numerical FT in Three Dimensions}

The procedure for obtaining the discrete three-dimensional transforms is completely similar to the previous cases and we quote only the final result here.

Assuming first that $F(r)$ vanishes for $r \geqslant R$, one obtains an ordinary Fourier sine expansion for $r F(r)$. With the additional assumption that $F\left(k_{j}\right)$ vanishes for $k_{s} \geqslant k_{N}=N \pi / R$, we get the discrete analogs of (6c)-(8c):

$$
\begin{gathered}
F\left(r_{i}\right)=\frac{1}{2 \pi^{2} r_{i}} \sum_{j=1}^{N-1} \Delta k k_{j} \tilde{F}\left(k_{j}\right) \sin k_{j} r_{i}, \\
\tilde{F}\left(k_{j}\right)=\frac{4 \pi}{k_{j}} \sum_{i=1}^{N-1} \Delta r r_{i} F\left(r_{i}\right) \sin k_{j} r_{i}, \\
\sum_{j=1}^{N-1} \Delta k \sin k_{j} r_{i} \sin k_{j} r_{\ell}=\frac{\pi}{2} \frac{\delta_{i \ell}}{\Delta r},
\end{gathered}
$$

with

$$
\begin{array}{rlrl}
r_{i} & =i \Delta r, & \Delta r & =R / N, \\
k_{j}=j \Delta k, & \Delta k & =\pi / R .
\end{array}
$$

Equation (62) is proved in Appendix C.

\section{A Numerical Test}

In the preceding three sections, orthogonality-preserving algorithms for the numerical calculation of Fourier transforms in one, two, and three dimensions 
have been obtained, the result being in each case similar to a trapezoidal rule with the intervals determined by the roots of $\cos x, J_{0}(x)$, and $\sin x$, respectively. In this section, we present the results of a numerical test of these algorithms.

For the test function, we choose a Gaussian,

$$
F(r)=\exp \left(-\frac{1}{2} r^{2}\right)
$$

for which the transforms are also Gaussian; in $d$ dimensions,

$$
\tilde{F}(k)=(2 \pi)^{d / 2} \exp \left(-\frac{1}{2} k^{2}\right) .
$$

The FT of (65) was evaluated numerically for $d=1,2$, and 3 using the schemes described above. A comparison of the numerical and exact transforms then affords a direct test of the accuracy of these quadrature rules. The results are shown in Table $\mathrm{I}$, in the form of the largest difference $(2 \pi)^{-d / 2}\left|\tilde{F}\left(k_{j}\right)^{\mathrm{ex}}-\tilde{F}\left(k_{j}\right)^{\text {comp }}\right|$, for calculations using $N=20,100$, and 200 intervals. In all cases $R$ was about 10 .

TABLE I

The Largest Difference $(2 / \pi)^{-d / 2}\left|\tilde{F}\left(k_{j}\right)^{e x}-\tilde{F}\left(k_{j}\right)^{\text {eamp }}\right|$ Between the Exact and Computed (Using

\begin{tabular}{|c|c|c|c|}
\hline \multirow[b]{2}{*}{$d$} & \multicolumn{3}{|c|}{$N$} \\
\hline & 20 & 100 & 200 \\
\hline 1 & $3.4 \times 10^{-10}$ & $7.0 \times 10^{-16}$ & $1.0 \times 10^{-14}$ \\
\hline $2^{a}$ & $1.9 \times 10^{-8}$ & $4.4 \times 10^{-8}$ & $5.8 \times 10^{-5}$ \\
\hline 3 & $2.7 \times 10^{-9}$ & $2.0 \times 10^{-15}$ & $2.0 \times 10^{-15}$ \\
\hline
\end{tabular}
$N$ Intervals) Gaussian Transforms in $d$ Dimensions

${ }^{a}$ The $d=2$ results are of the order of the error in the computed Bessel functions.

The $d=1$ and 3 cases were computed in double precision on an IBM 360, Model 75 , for which machine accuracy is 16 to 17 decimal digits. It is evident from Table I that the numerical FT in these cases are essentially exact for $N=100$ and 200. The two-dimensional case was evaluated in single precision on a CDC 6600 , which provides 14-15 significant decimal digits. However, the error in the computed roots and values of $J_{0}$ was of the order of $10^{-8}$, so that the computed FT in the two-dimensional case is, from Table I, again essentially exact, within the accuracy obtainable [8]. As expected, the numerical inversion of the computed transforms reproduces, in all cases, the original function to about the accuracy of the computed basis functions.

The two- and three-dimensional algorithms have in addition been used in the numerical solution of integral equations for hard-core fluids $[9,10]$, with satisfactory results, 


\section{CONCLUSIONS}

In situations where a Fourier transform is to be numerically computed and subsequently numerically inverted, perhaps repetitively, it is desirable that the computational algorithm used to accomplish this maintain the orthogonality of the discrete basis functions appearing in the numerical transforms. Algorithms with this property have been obtained above for the FT of central functions in one, two, and three dimensions. These algorithms are constituted by Eqs.(26)-(30), (46)-(50), and (60)-(64), respectively. A numerical check using a Gaussian test function has shown that these rules are accurate as well. The algorithms for twoand three-dimensional transforms have in addition been successfully applied to the calculation of the radial distribution function for hard-core systems $[9,10]$.

Finally, the procedure followed in obtaining these results is applicable to other transforms as well, such as forms of the Fourier-Bessel transform involving Bessel functions other than $J_{0}$.

\section{APPENDIX A. Discrete Orthogonality Condition for Numerical FT in ONE DIMENSION}

In this appendix, we prove the orthogonality condition used in Section II in arriving at the discrete Fourier transform in one dimension. We need to evaluate the sum

$$
\begin{aligned}
D_{\ell j}^{(1)} & =\sum_{n=1}^{N} \cos k_{n} r_{\ell} \cos k_{n} r_{j} \\
& =\frac{1}{2} \sum_{n=1}^{N}\left\{\cos k_{n}\left(r_{\ell}-r_{j}\right)+\cos k_{n}\left(r_{\ell}+r_{j}\right)\right\} \\
& =\frac{1}{2} \sum_{n=1}^{N}\left\{\cos \frac{(2 n-1)(\ell-j) \pi}{2 N-1}+\cos \frac{(2 n-1)(\ell+j-1) \pi}{2 N-1}\right\},
\end{aligned}
$$

where Eqs. (29) and (30) have been used. The sum of cosines in (A1) is [11]

$$
\sum_{n=+}^{N} \cos (2 n-1) \theta=\frac{1}{2} \sin 2 N \theta \csc \theta,
$$


so that (A1) becomes

$$
\begin{aligned}
D_{\ell j}^{(1)}= & \frac{1}{4}\left\{\sin \frac{2 N(\ell-j) \pi}{2 N-1} \csc \frac{(\ell-j) \pi}{2 N-1}\right. \\
& \left.+\sin \frac{2 N(\ell+j-1) \pi}{2 N-1} \csc \frac{(\ell+j-1) \pi}{2 N-1}\right\} \\
= & \frac{1}{4}\left\{\sin (\ell-j) \pi \csc \frac{(\ell-j) \pi}{2 N-1} \cos \frac{(\ell-j) \pi}{2 N-1}+\cos (\ell-j) \pi\right. \\
& +\sin (\ell+j-1) \pi \csc \frac{(\ell+j-1) \pi}{2 N-1} \cos \frac{(\ell+j-1) \pi}{2 N-1} \\
& +\cos (\ell+j-1) \pi\} \\
= & \frac{1}{4} \sin (\ell-j) \pi \csc \frac{(\ell-j) \pi}{2 N-1} \cos \frac{(\ell-j) \pi}{2 N-1} .
\end{aligned}
$$

This last expression also vanishes if $l \neq j$. But as $l$ approaches $j$, it has the limiting value $(2 N-1) / 4$. Thus we get

$$
D_{\ell j}^{(1)}=\frac{1}{4}(2 N-1) \delta_{\ell j},
$$

which is Eq. (24).

APPENDIX B: Discrete ORThOGONALITY CONDITION FOR NUMERICAL FT in Two Dimensions

The proof of Eq. (45) for transforms in two dimensions is more involved than that of the corresponding cases in one and three dimensions, and, in the end, less general. Briefly, the proof consists in expressing the sum of Bessel functions in (45) in terms of a contour integral in the complex plane, and then evaluating the integral. A similar proof could be made for the one- and three-dimensional cases.

The following proof is adapted from Gray, Mathews, and Macrobert [12]. Consider the function

$$
Q(z)=\frac{z H_{0}^{(1)}(z) J_{0}(s z) J_{0}(t z)}{J_{0}(z)}
$$

where $H_{0}^{(1)}(z)$ is the Hankel function

$$
H_{0}^{(1)}(z)=J_{0}(z)+i Y_{0}(z),
$$


$Y_{0}(z)$ being the zeroth-order Bessel function of the second kind, and where

$$
\begin{aligned}
s & \equiv \mu_{6} / \mu_{N} \\
t & \equiv \mu_{j} / \mu_{N} .
\end{aligned}
$$

The zeros of $J_{0}(z)$ are simple poles of $Q(z)$; the residue at $z= \pm \mu_{n}$ is

$$
\begin{aligned}
\lim _{z \rightarrow \pm \mu_{n}}\left(z \mp \mu_{n}\right) Q(z) & =\frac{\mu_{n} H_{0}^{(1)}\left(\mu_{n}\right) J_{0}\left(s \mu_{n}\right) J_{0}\left(t \mu_{n}\right)}{J_{0}^{\prime}\left(\mu_{n}\right)} \\
& =-\frac{2 i}{\pi} \frac{J_{0}\left(s \mu_{n}\right) J_{0}\left(t \mu_{n}\right)}{\left[J_{0}^{\prime}\left(\mu_{n}\right)\right]^{2}},
\end{aligned}
$$

where we have used the fact that

$$
\lim _{z \rightarrow \pm \mu_{n}} \frac{z \mp \mu_{n}}{J_{0}(z)}= \pm \frac{1}{J_{0}^{\prime}\left(\mu_{n}\right)}
$$

The second equality in (B5) follows from (B2) and the Wronskian [13]

$$
J_{0}(z) Y_{0}^{\prime}(z)-J_{0}^{\prime}(z) Y_{0}(z)=2 / \pi z
$$

evaluated at the root $z=\mu_{n}$. In addition, $Q(z)$ has a logarithmic singularity at the origin due to $H_{0}^{(1)}(z)$, with zero residue.

Note that (B5) contains just the summand desired. Consider now the integral of $Q(z)$ around the contour $C$ shown in Fig. 1; since $Q(z)$ has a logarithmic branch point at the origin, the complex plane is cut along the negative imaginary axis. All singularities are excluded from the interior of $C$, so that we have

$$
\int_{C} Q(z) d z=0
$$

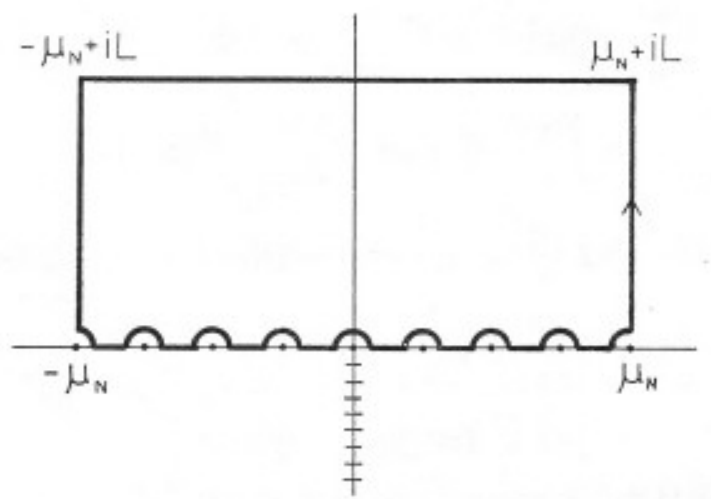

FIG, 1. Contour for the integral of $Q(z)$. Cross-hatching denotes a branch cut. 
Let us now evaluate the integral along each of the segments of $C$. With (B5), the contribution of the semicircular paths about the singularities, in the limit of vanishing radii, is found to be $-4 D_{\ell j}^{(2)}$, where

$$
\begin{aligned}
D_{l j}^{(2)} & =\sum_{n=1}^{N-1} \frac{J_{0}\left(\mu_{n} \mu_{\ell} / \mu_{N}\right) J_{0}\left(\mu_{n} \mu_{j} / \mu_{N}\right)}{\left[J_{0}^{\prime}\left(\mu_{n}\right)\right]^{2}} \\
& =\sum_{n=1}^{N-1} \frac{J_{0}\left(k_{n} r_{\ell}\right) J_{0}\left(k_{n} r_{j}\right)}{\left[J_{0}^{\prime}\left(k_{n} R\right)\right]^{2}} .
\end{aligned}
$$

Also in the limit of vanishing radii, the straight line segments along the real axis yield

$$
\begin{aligned}
& P \int_{-\mu_{N}}^{0} Q(x) d x+P \int_{0}^{\mu_{N}} Q(x) d x \\
& \quad=P \int_{0}^{\mu_{N}}\left\{Q\left(x e^{i \pi}\right)+Q(x)\right\} d x \\
& \quad=2 \int_{0}^{\mu_{N}} x J_{0}\left(\mu_{\ell} x / \mu_{N}\right) J_{0}\left(\mu_{j} x / \mu_{N}\right) d x \\
& =\mu_{N}{ }^{2}\left[J_{0}^{\prime}\left(\mu_{j}\right)\right]^{2} \delta_{\ell j} .
\end{aligned}
$$

Here $P$ means Cauchy principal value and we have used the analytic continuations of $J_{0}(z)$ and $H_{0}^{(1)}(z)$ to obtain the second line of (B10). Letting $L$ go to infinity, one can show, using the asymptotic forms of $J_{0}(z)$ and $H_{0}^{(1)}(z)$, that $Q(z)$ vanishes exponentially along the upper horizontal branch of $C$ if $\mu_{t}+\mu_{j}<2 \mu_{N}$. Finally, the integrals along the vertical branches can be written, in the limit $L \rightarrow \infty$,

$$
\begin{aligned}
\int_{u_{N}}^{u_{N}+i \infty} & Q(z) d z+\int_{-\mu_{N}+i \infty}^{\mu_{N}} Q(z) d z \\
= & \int_{u_{N}}^{u_{N}+i \infty} Q(z) d z-\int_{\mu_{N}-i \infty}^{\mu_{N}} Q\left(z e^{i \pi}\right) d z \\
= & i \int_{0}^{\infty}\left\{Q\left(\mu_{N}+i y\right)-Q\left[\left(\mu_{N}-i y\right) e^{i \pi}\right]\right\} d y \\
= & i \int_{0}^{\infty}\left\{Q\left(\mu_{N}+i y\right)-Q\left(\mu_{N}+i y\right)^{*}\right\} d y \\
= & -2 \int_{0}^{\infty} \operatorname{Im}\left[Q\left(\mu_{N}+i y\right)\right] d y \\
= & -\mu_{N}{ }^{2}\left[J_{0}^{\prime}\left(\mu_{j}\right)\right]^{2} \epsilon_{\ell j}^{(N)},
\end{aligned}
$$


where the last equality defines $\epsilon_{\ell_{j}}^{(N)}$. Here the asterisk denotes complex conjugate, $\operatorname{Im}(A)$ is the imaginary part of $A$, and the third line in (B11) follows again from the analytic properties of $J_{0}(z)$ and $H_{0}^{(1)}(z)$.

Collecting these partial results into (B8), we have finally

$$
-4 D_{\ell j}^{(2)}+\mu_{N}{ }^{2}\left[J_{0}^{\prime}\left(\mu_{j}\right)\right]^{2}\left\{\delta_{\ell j}-\epsilon_{\ell j}^{(N)}\right\}=0,
$$

or

$$
D_{\ell j}^{(2)}=\frac{1}{4} \mu_{N}{ }^{2}\left[J_{0}{ }^{\prime}\left(\mu_{j}\right)\right]^{2}\left\{\delta_{\ell j}-\epsilon_{\ell j}^{(N)}\right\} .
$$

It remains to be shown that $\epsilon_{\ell j}^{(N)}$ vanishes, which, from (B11), evidently follows if $Q\left(\mu_{N}+i y\right)$ is real. This is easily shown to be the case for large $N$, when, using the asymptotic forms of $J_{0}(z)$ and $H_{0}^{(1)}(z)$, we find that

$$
Q(z) \sim \frac{2(-1)^{6+j}}{\pi(s t)^{1 / 2}} \frac{\sinh s y \sinh t y}{\sinh y} e^{-y},
$$

thus establishing (45) at least asymptotically. We have not succeeded in showing that $\epsilon_{b j}^{(N)}$ vanishes in general. However, Eq. (45) is better than the present argument may suggest. A direct numerical evaluation of the sum in $D_{b j}^{(2)}$ shows [10] that $\left|\epsilon_{\ell j}^{(N)}\right|$ is (for all $\ell, j$ ) already of order $10^{-6}$ for $N=5$, and of the order of the error in the computed Bessel functions, about $10^{-8}$, for $N=20$. Since, in numerical calculations, $N$ will generally be several hundred, Eq. (45) follows as a numerically correct orthogonality condition for such cases.

\section{APPENDIX C: Discrete Orthogonality CONDItion fOR Numerical FT in} Three Dimensions

The evaluation of

$$
D_{\ell j}^{(3)}=\sum_{n=1}^{N} \sin k_{n} r_{\ell} \sin k_{n} r_{j}
$$

is similar to that of $D_{l j}^{(1)}$. Using a trigonometric identity and Eqs. (63) and (64), we have

$$
\begin{aligned}
D_{\ell j}^{(3)} & =\frac{1}{2} \sum_{n=1}^{N}\left\{\cos k_{n}\left(r_{\ell}-r_{j}\right)-\cos k_{n}\left(r_{\ell}+r_{j}\right)\right\} \\
& =\frac{1}{2} \sum_{n=1}^{N}\{\cos n(\ell-j) \pi / N-\cos n(\ell+j) \pi / N\} .
\end{aligned}
$$


The sum over cosines is here [11]

$$
\sum_{n=1}^{N} \cos n \theta=\sin \frac{1}{2} N \theta \cos \frac{1}{2} N \theta \cot \frac{1}{2} \theta-\sin ^{2} \frac{1}{2} N \theta
$$

so that $(\mathrm{C} 2)$ becomes

$$
\begin{aligned}
D_{\ell j}^{(3)}= & \frac{1}{2}\left\{\sin (\ell-j) \frac{\pi}{2} \cos (\ell-j) \frac{\pi}{2} \cot (\ell-j) \frac{\pi}{2 N}-\sin ^{2}(\ell-j) \frac{\pi}{2}\right. \\
& \left.-\sin (\ell+j) \frac{\pi}{2} \cos (\ell+j) \frac{\pi}{2} \cot (\ell+j) \frac{\pi}{2 N}+\sin ^{2}(\ell+j) \frac{\pi}{2}\right\} \\
= & \frac{1}{2} \sin (\ell-j) \frac{\pi}{2} \cos (\ell-j) \frac{\pi}{2} \cot (\ell-j) \frac{\pi}{2 N} \\
= & \frac{1}{2} N \delta_{\ell j},
\end{aligned}
$$

which is Eq. (62).

\section{ACKNOWLEDGMENT}

The author is indebted to Professor A. A. Broyles for the original motivation, and initiation into the problem, which led to this work.

\section{REFERENCES}

1. "Handbook of Mathematical Functions" (M. Abramowitz and I. A. Stegun, Eds.), Appl. Math. Ser., Vol. 55, p. 360, U. S. Govt. Printing Office, Washington, D. C. 1964.

2. L. N. G. Filon, Proc. Roy. Soc. Edinburgh Sect. $A 49$ (1928), 38; see also Ref. 1, p. 890.

3. G. C. Danielson and C. Lanczos, J. Franklin Inst. 233 (1942), 365 and 435. An earlier version of this idea may be found in R. W. Prentiss, Phys. Rev. 15 (1902), 257.

4. J. W. COOLEY AND J. W. TuKeY, Math. Comp. 19 (1965), 297. This algorithm has recently been applied to the radial distribution function by A. J. StarshaK and R. D. Larsen, Phys. and Chem. of Liquids 2 (1970), 45.

5. Ref. 1, p. 485.

6. A. Gray, G. B. Mathews and T. M. Macrobert, "A Treatise on Bessel Functions," 2nd edition, Chap. 8, MacMillan and Co., London, 1952.

7. Ref. 1, pp. 364 and 371.

8. The author is indebted to a reviewer for pointing out the following two references which should be useful in improving this accuracy: W. KAHAN and J. VARAH, "Two Working Algorithms for the Eigenvalues of a Symmetric Tridiagonal Matrix," Tech. Rept. No. CS43, Computer Science Dept., Stanford Univ., 1966; H. Gerber, Math. Comp. 18 (1964), 319. 
9. F. Lado, J. Chem. Phys. 47 (1967), 4828.

10. F. Lado, J. Chem. Phys. 49 (1968), 3092.

11. L. B. W. Jolley, "Summation of Series," 2nd revised edition, p. 78, Dover, New York, 1961

12. Ref. 6 , p. 95 .

13. A convenient summary of this and other pronerties of Bessel functions used in this appendix may be found in Ref. 1, Chap. 9.

Printed in Belgium by the St. Catherine Press Ltd., Tempelhof 37 , Bruges 